\title{
A smarter way to treat obesity
}

Although chronic inflammation has been implicated in the pathogenesis of diet-induced obesity (DIO), the anti-obesity potential of anti-inflammatory drugs (such as the glucocorticoid dexamethasone; Dexa) has been hampered by off-target adverse effects and limited potency.

In potentially breakthrough research reported in Cell Metabolism, a team led by Brian Finan and Matthias Tschöp show that precision targeting of Dexa to cells expressing the glucagon-like peptide 1 receptor (GLP1R) markedly reduces obesity and reverses immunometabolic dysfunction without the hallmark adverse effects of chronic Dexa treatment. The approach, which combines incretin and glucocorticoid pharmacology, could pave the way for cell-selective, anti-inflammatory metabolic 'smart drugs' being used to treat metabolic inflammation and obesity.

As GLP1R is expressed in the hypothalamus and pancreas (among other tissues), and in peripherally active immune cells, the investigators envisioned that a GLP1-Dexa co-agonist might selectively attenuate both hypothalamic and systemic inflammation, and the resulting obesity. After testing a series of GLP1-Dexa conjugates with different linkers, the team selected a conjugate with a biometabolizable linker (capable of releasing native Dexa under reducing conditions that mimic the intracellular environment) for in vivo studies.

Mice with DIO treated with the GLP1-Dexa co-agonist displayed marked reductions in body weight (25\% reduction from baseline), adiposity and food intake, and increased energy expenditure. Moreover, co-agonist treatment reversed the glycaemic complications associated with obesity in mice with DIO and reduced both hypothalamic and systemic inflammation. Crucially, these immunometabolic improvements were achieved without inducing the adverse effects associated with

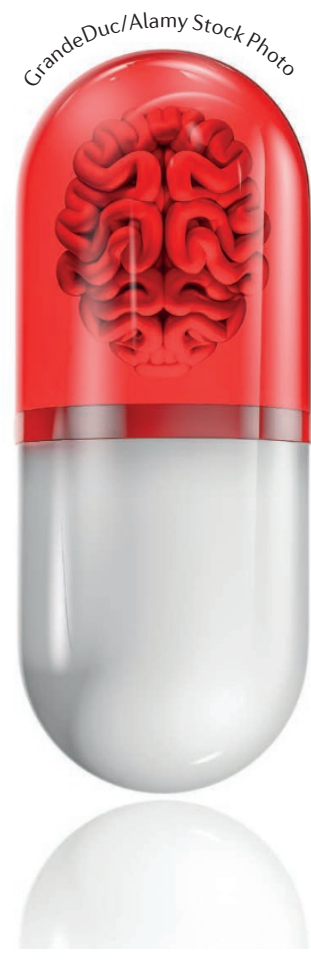

chronic Dexa treatment (such as hyperglycaemia, osteoporosis and derangement of the hypothalamicpituitary-adrenal axis).

"We have developed a novel pharmacological strategy that enables safe, precise targeting of the immunometabolic alterations induced by hypercaloric diets, while circumventing many of the off-target liabilities of Dexa," comment Finan, Tschöp and first author Carmelo Quarta. "Although clinical trials have to prove that this concept can be translated to humans, our discovery firmly positions commonly used steroid-based medications such as Dexa as candidate precision therapeutics for improving metabolic inflammation and associated sequelae."

David Holmes

ORIGINAL ARTICLE Quarta, C. et al. Molecular integration of incretin and glucocorticoid action reverses immunometabolic dysfunction and obesity. Cell Metab. http://dx.doi.org/10.1016/j. cmet.2017.08.023 (2017) 